\title{
Striving for more good days: patient perspectives on botulinum toxin for the treatment of cervical dystonia
}

\author{
Michele Poliziani' \\ Marco Koch ${ }^{2}$ \\ Xierong Liu' \\ 'Opinion Health, London, UK; ${ }^{2}$ Merz \\ Pharmaceuticals $\mathrm{GmbH}$, Frankfurt am \\ Main, Germany
}

This article was published in the following Dove Press journal:

Patient Preference and Adherence

22 August 2016

Number of times this article has been viewed

Background: The recommended reinjection interval for botulinum neurotoxin (BoNT) formulations in the treatment of cervical dystonia (CD) is generally $\geq 12$ weeks, though intervals $\geq 10$ weeks are approved for incobotulinumtoxinA in Europe. However, recurring symptoms can occur before the end of this period. Using qualitative research, we sought a greater understanding of disease burden, unmet patient needs, and barriers to treatment.

Methods: We conducted online semistructured, focus-group discussions, and online forum follow-up discussions among patients with $\mathrm{CD}$, focusing on disease burden, patient needs, injection cycle preferences, and relationships with health care professionals. A subset of patients was also questioned in telephone interviews about individual experiences of CD and BoNT treatment. All participants were UK residents who had received onabotulinumtoxinA or abobotulinumtoxinA for CD for $\geq 1$ year.

Results: Thirty-one patients ( $81 \%$ female; mean duration of CD 16.4 [range 4-31] years; mean BoNT injection cycle length 12.8 weeks) participated in the online focus-group and forum follow-up discussions. Of these, seven patients participated in telephone interviews. All had recurring symptoms between treatments, which substantially impacted on their work, family, and social life. Symptom severity fluctuated throughout an injection cycle and differed between patients and across injection cycles. Participants' relationships with health care professionals and treatment satisfaction varied greatly. Many participants wanted longer-lasting and/or more stable symptom relief with shorter and/or more flexible injection intervals, according to individual needs. Lack of health care resources, long journeys to treatment centers, and immunogenicity/ side-effect concerns were perceived as the main barriers to more flexible treatment.

Conclusion: The high burden of recurring primary and secondary symptoms of CD considerably affects patients' quality of life. Patient-led assessments of disease burden revealed that personalized, more flexible, and/or shorter BoNT injection intervals may reduce the day-to-day impact of CD. Collaboration between patients, clinicians, and health care systems may effect change and improve treatment for patients with $\mathrm{CD}$.

Keywords: botulinum toxin type A, cervical dystonia, patient survey, spasmodic torticollis, treatment satisfaction

\section{Introduction}

Cervical dystonia (CD), also known as spasmodic torticollis, is the most common focal dystonia and is characterized by contraction of the cervical muscles leading to abnormal head, neck, and shoulder posture, as well as head tremor. ${ }^{1,2}$ Although CD can occur at any age, symptoms usually become evident during the fifth decade, often beginning slowly and progressing to a plateau over a few months or years. ${ }^{1,2}$ Approximately one in 
ten patients may experience spontaneous remission, but this may not be permanent. ${ }^{2} \mathrm{CD}$ is a painful condition that reduces quality of life (QoL), leading to anxiety and depression. ${ }^{3-5}$ It also has a negative impact on employment, for example, reducing an individual's productivity, their ability to work certain hours, or perform responsibilities, and may lead to loss of employment. ${ }^{6}$

The recommended treatment for $\mathrm{CD}$ is repeated injection of botulinum neurotoxin (BoNT), ${ }^{7-9}$ and the clinical efficacy of BoNT in this setting has been confirmed in a number of randomized, double-blind clinical trials. ${ }^{10-12}$ The duration of action of BoNT is reported to range between $\sim 9.5$ and 16 weeks. ${ }^{13-16}$ However, in one study the therapeutic effect of a BoNT injection lasted $\leq 10$ weeks in $36 \%$ of patients treated. ${ }^{16}$ In the UK, the minimum recommended injection interval for the treatment of $\mathrm{CD}$ with onabotulinumtoxinA (Botox; Allergan Inc., Parsippany, NJ, USA) or abobotulinumtoxinA (Dysport; Ipsen Ltd, Slough, Berkshire, UK) is 12 weeks, ${ }^{17,18}$ though intervals $\geq 10$ weeks are approved for incobotulinumtoxinA (Xeomin; Merz Pharmaceuticals $\mathrm{GmbH}$, Frankfurt/Main, Germany) because it is free from complexing (accessory) proteins. ${ }^{19}$

Earlier reinjection is not recommended because of concerns that shorter intervals may increase the risk of side effects and generation of an immune response to treatment. ${ }^{20}$ However, it is evident that the actual interval between BoNT injections can be longer than the duration of action, ${ }^{13,16}$ leaving patients with suboptimal symptom relief toward the end of the treatment cycle. Newer treatment guidelines recognize that an injection schedule with fixed intervals of 12 weeks may not be appropriate for all patients and suggest adopting a flexible dosing schedule according to clinical response. ${ }^{21}$

Clinical studies have demonstrated that BoNT treatment improves QoL in patients with CD. ${ }^{3,5,21,22}$ However in these studies, QoL was typically assessed at defined time points during the injection cycle - most commonly before an injection and 4 weeks later, when the assumed peak effect of BoNT treatment occurred. ${ }^{5,22}$ Little is known about the impact of CD on different aspects of individual patients' lives during the waning period (ie, the period of time when BoNT treatment effects start to decline but before patients undergo reinjection), particularly in a real-life setting. A recent study of patients with CD who received BoNT treatment and who experienced recurring symptoms between injection cycles revealed that the aspects most commonly influenced by symptoms are stigma, pain, and activities of daily living, although emotional well-being and social/family life are also impacted, with negative consequence on leisure activities, work life, and mood. ${ }^{23}$
BoNT injections provide many patients with effective relief from the often painful and debilitating effects of CD. ${ }^{24}$ There is, nevertheless, scope for further improvements in therapy and outcomes for patients with CD. These improvements are likely to stem from a more thorough understanding of the condition and the individual needs of affected patients. We conducted a qualitative study with the intention of gaining a greater understanding of the impact of BoNT injections that would provide a comprehensive and emotional picture of patients with $\mathrm{CD}$, their preferences in relation to BoNT injection cycles (with particular focus on the waning period), their relationships with health care professionals, the burden of disease, and the potential unmet needs of treatment.

\section{Methods \\ Study design}

This qualitative-exploratory research was conducted in the UK and was based on three complementary methods: 1) an online focus-group discussion, 2) a subsequent online forum follow-up discussion, and 3) individual telephone interviews.

The focus-group participated in a 60-minute, semistructured online discussion. All participants were invited to contribute to subsequent online forum follow-up discussions, which ran asynchronously over 2 weeks. In the individual 30-minute telephone interviews, patients from the online focus-group were questioned in depth about their individual experiences with $\mathrm{CD}$ and BoNT treatment for CD.

Explicit consent for each participant was collected via an online link at the time of recruitment, during which all respondents were made aware of their involvement and the scope of the research. Ethics Committee and Independent Review Board approval was not required for this research based on the Code of Conduct of the European Pharmaceutical Market Research Association (EphMRA), as the information gathered was for market research purposes only; no invasive research, human testing, or access to patient records were required in the recruitment or data collection process.

The focus-group was set up specifically for the study. Some participants for the telephone interview were selected according to the follow-up questions that were raised from their responses during the focus-group discussion, while the others were invited because their responses during the focus-group discussion were considered to be particularly representative or insightful.

\section{Participant recruitment}

Patients with CD completed a 5-minute online profiling questionnaire to evaluate eligibility for the study. Patients 
with a confirmed diagnosis of $\mathrm{CD}$ were included in the study if they had received injections of onabotulinumtoxinA or abobotulinumtoxinA for the treatment of $\mathrm{CD}$ for $\geq 1$ year. Patients who had received incobotulinumtoxinA were excluded since the minimum recommended interval for this formulation in the UK is 10 weeks. ${ }^{19}$ Patients were required to have experienced at least some recurrence of symptoms between injections.

\section{Data collection}

Online focus-group discussions and online forum follow-up discussions

A 60-minute online focus-group discussion was led by a moderator, employed by Opinion Health (London, UK), who was supplied with guidance on specific questions in order to facilitate discussions. The discussion guide included unbiased, open-ended questions on the course of symptom development during a typical injection cycle, disease burden and QoL, patient-doctor communication, and patient needs and preferences, including, for example: "How often do you receive these injections?", "Could you describe how these injections change your experience of the symptoms?", "What words would you use to describe the emotions you experience when the symptoms start to reappear after the injection?", and "If you could freely choose, how often would you like to receive your injections?"

In addition, all participants were invited to contribute to an online forum follow-up discussion in which they were asked to comment on their experience of CD symptoms, how these affected their life and their relationships with other people, including family members and doctors, the impact of BoNT treatment on CD symptoms, and their expectations of treatment. The frequency and severity of symptoms that occurred between injections were explored by asking participants to choose one of four predefined patterns, represented graphically, according to the degree of symptom relief achieved with BoNT injections and whether the pattern remained consistent within or between injection cycles.

\section{Telephone interviews}

A moderator employed by Opinion Health led individual 30-minute telephone interviews with participants of the online focus-group and online forum follow-up discussions. The moderator followed a guide to facilitate discussions; this included questions on symptoms between injections, satisfaction with current treatment, role of health care professionals in CD treatment, and topics related to injections (side effects, interval between injections, and shorter/flexible intervals).
Audio recordings and transcriptions were made of all individual interviews.

\section{Data analysis}

The online focus-group discussions and online forum follow-up discussions, and responses contained in interview transcripts were examined in detail by the study analysts from Opinion Health, and the information was categorized into themes and concepts. These were considered according to the waning period following a BoNT injection and the patients' personal perspectives on symptom recurrence, the control of symptoms during the relief period following a BoNT injection and patient expectations from treatment, and finally, the patient-health care professional relationship. Responses were subjected to content analyses.

\section{Results \\ Participants}

Research was conducted between June 26 and July 31, 2013. A total of 31 patients with CD were enrolled in the study and participated in the online focus-group discussions and online forum follow-up discussions. Of these, seven were selected for telephone interviews. The majority of participants were female $(81 \%)$ and aged $45-54$ years $(41.9 \%)$. The mean duration of CD was 16.4 years (range 4-31 years); the mean length of the patients' BoNT injection cycle was 12.8 weeks (range 8-20 weeks) (Table 1).

\section{Waning period: recurrence of symptoms}

Although all patients who participated in the study experienced recurring CD symptoms between BoNT injections, symptom severity varied throughout an injection cycle and differed between patients and across injection cycles.

Table I Participants' demographics and characteristics

\begin{tabular}{ll}
\hline Demographic & $\begin{array}{l}\text { Survey participants } \\
(\mathbf{n}=\mathbf{3} \mathbf{I})\end{array}$ \\
\hline Female, $\mathrm{n}(\%)$ & $25(8 \mathrm{I})$ \\
Age group (years), $\mathrm{n}(\%)$ & \\
$25-34$ & $2(6.5)$ \\
$35-44$ & $8(25.8)$ \\
$45-54$ & $13(4 \mathrm{I} .9)$ \\
$55-64$ & $5(16.1)$ \\
$\geq 65$ & $3(9.7)$ \\
UK region, $\mathrm{n}(\%)$ & \\
England & $27(88)$ \\
Scotland & $2(6)$ \\
Northern Ireland & $\mathrm{I}(3)$ \\
Wales & $\mathrm{I}(3)$ \\
Mean (range) duration of CD, years & $16.4(4-3 \mathrm{I})$ \\
Mean (range) BoNT injection cycle length, weeks & $\mathrm{I} 2.8(8-20)$ \\
\hline
\end{tabular}

Abbreviations: BoNT, botulinum neurotoxin; $C D$, cervical dystonia. 
Throughout an injection cycle, symptoms were described as gradually returning or intensifying, but there was no clear pattern of symptom recurrence within each injection cycle. When we compared symptom recurrence between patients, for some, the symptoms returned following a set pattern during each injection cycle; although the order in which symptoms returned differed between patients; pain, tremor, or a pulling sensation was commonly the first symptom to return. For other patients, symptoms recurred with no set pattern.

CD symptoms could be categorized as core (reported by almost all patients, eg, pain, spasms, tremors, and pulling), primary (directly caused by $\mathrm{CD}$, eg, fatigue and loss of balance), and secondary (sequelae of core/primary symptoms, eg, falls and fractures, or broken teeth caused by jaws clamping shut) (Figure 1).

Of the 31 patients enrolled, 25 were able to categorize their typical symptom progression into one of four different patterns, based on graphical representations in the online research (Figure 2). Pattern 4 (an inverted bell shape, with a considerable level of day-to-day symptom fluctuation) was most frequently chosen $(n=12)$.

\section{Waning period: QoL}

When asked to illustrate the impact of recurring symptoms on their lives and families, patients described how CD symptoms reduced their QoL. Participants stated that their symptoms stopped them from pursuing their careers and damaged their confidence to return to work, placed a heavy burden on themselves and others around them, and caused isolation and alienation as they felt they could no longer enjoy an active and healthy social life owing to the social stigma they experienced. As a consequence, patients reported reduced emotional well-being, with feelings of depression, embarrassment, and frustration.

\section{Expectations from BoNT treatment}

Most patients rarely experienced any symptom-free days. In general, CD symptoms were still present to some extent even during the relief period, when BoNT treatment effects were felt at their strongest. Nevertheless, patients highly valued any period of relief from symptoms, and acknowledged that BoNT treatment enabled them to have "more good days" by lowering the impact of CD on their lives.

\section{BoNT injection and the relief period: optimization of treatment between injections}

When asked about "their ideal injection cycle", participants wanted longer-lasting and/or more stable symptom relief with shorter and/or more flexible injection intervals in accordance with the individual needs of the patient (Table 2). Staff and resource shortages, long journey times to treatment

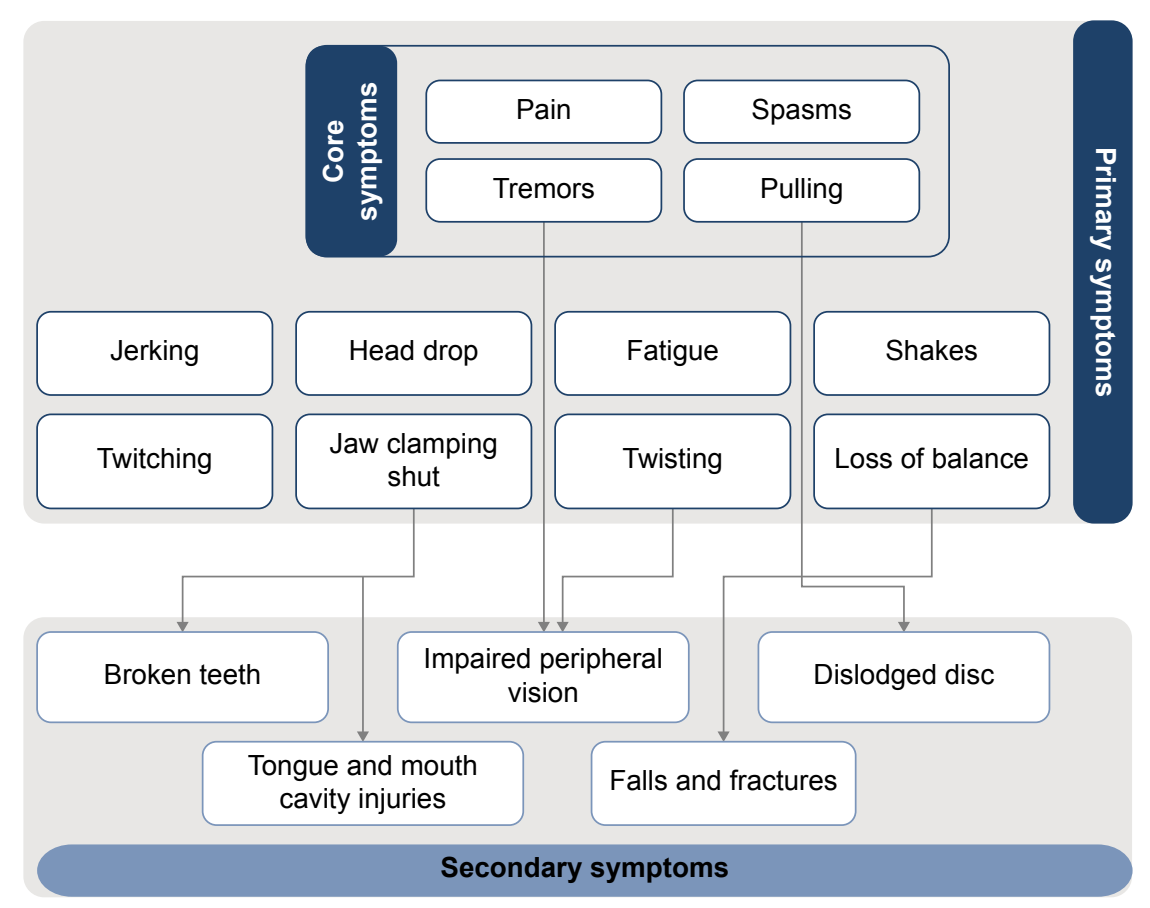

Figure I Conceptual representation of recurring core, primary, and secondary symptoms in cervical dystonia.

Notes: Core symptoms are those reported by almost all participants. Primary symptoms are those directly associated with cervical dystonia. Secondary symptoms are those caused by the primary symptoms of cervical dystonia. 


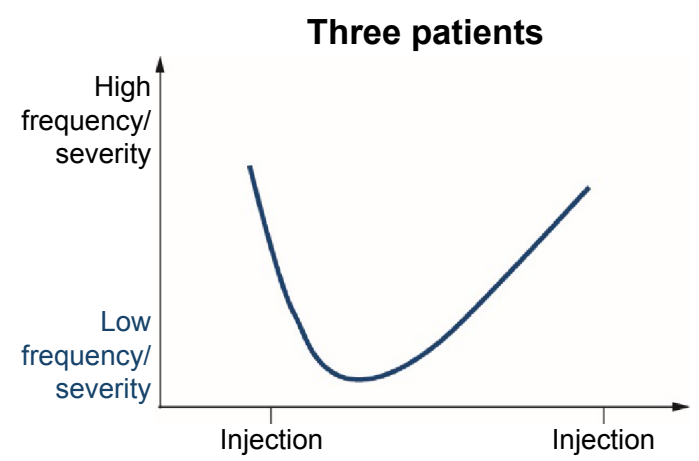

Six patients

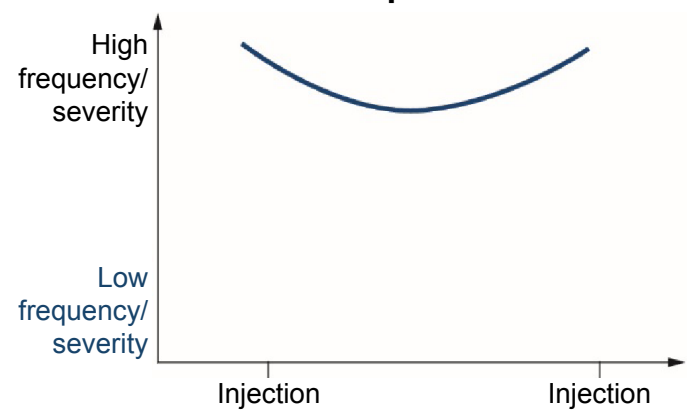

\section{Four patients}

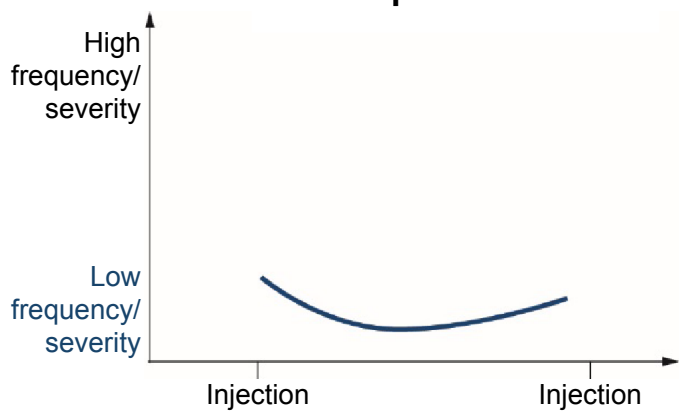

Twelve patients

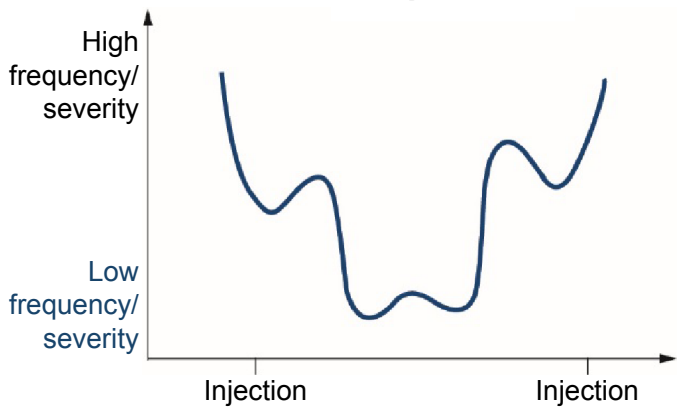

Figure 2 Patterns of symptom progression between botulinum neurotoxin injections.

centers, and concerns about side effects were perceived to be the main barriers to shorter/more flexible injection intervals (Table 2).

\section{Relationships with health care professionals}

Patients' relationships with health care professionals and their satisfaction with the level of care they received varied widely.
Awareness of CD among primary care professionals was reported to be low, and patients received no support or care from their primary care physician; instead, their chief role was to refer patients for secondary care. As such, participants did not visit their primary care physician about $C D$ beyond the initial referral and their condition was managed in the secondary care setting. In the secondary care setting, some participants felt that health care professionals had little time

Table 2 Themes identified as being related to shorter/flexible injection intervals (verbatims)

\section{Shorter injection cycles}

"Every 8 weeks cause that's when the effect wears off and the pain starts to become unbearable".

"As soon as they start to wear off, ie, around every 6 weeks".

"Every 6-8 weeks would be nice :-)".

"I would, if I could, choose to have my injections every 6 weeks but I know that's not possible".

Flexible injection cycles

"Would feel more stable/in control if injections flexible, life would be a bit more predictable instead of a constant roller coaster of symptoms".

"More accessible, flexible treatment. If you have the flu you don't have to live with your symptoms for 6 weeks before your doc will see you".

"... it being available to everyone, no matter where they live and on a regular basis when they need it".

"It would be great if I could get the injection whenever I need it".

\section{Barriers to shorter/more flexible injection cycles}

Staff and resource shortage: "I find it too stressful to call for a doc's appointment ... wouldn't like the stress of calling and not getting an appointment for a few weeks".

"They (the hospital) don't have a nice friendly secretary who can arrange an appointment; it's done by the 'appointments department' so therefore no understanding of my condition".

"I once had to go 6 months (before re-injection because of) staff shortages".

"The neurologist is always too busy to do more than administer the injections".

Journey times: "I hate the long journey for my appointments, and my husband has to take time off work".

"I have to go to another hospital approx. 25-30 miles away".

Side effects: "Personally, l'd be worried about muscle wastage (which l've already had) and increased side effects".

"I've been told that they won't inject before the 13 weeks is up to stop you from becoming immune to it". 
Table 3 The health economic impact of recurring symptoms of cervical dystonia

\section{Impact on patient and family}

Reduced emotional well-being of patients and families (eg, reduced leisure activities)

Lost work hours relating to the carer (eg, driving patients to the clinic)

Lost work hours relating to the patient (eg, sick leave)

Provision of informal care by family members

Impact on society

Cost to the health care system (eg, provision of alternative treatment) Cost to the welfare system (eg, benefits, unemployment)

to get to know them or become familiar with their patient history. However, other participants reported good long-term relationships with health care professionals in the secondary care setting.

\section{Discussion}

This nonconventional, qualitative-exploratory study highlights the significant impact of recurring symptoms between BoNT injection cycles, which negatively affect the QoL and emotional well-being of patients with $\mathrm{CD}$. An additional consequence is a substantial health economic burden, involving costs associated with lost working hours, as well as expenses pertaining to the welfare system and health care providers (Table 3). The most fundamental frustration identified by patients was the limiting impact of $\mathrm{CD}$ on their ability to carry out everyday activities.

Our findings provide a real-life view of patients' perspectives of the impact of $\mathrm{CD}$ and BoNT injection treatment on their daily lives throughout an injection cycle - during the relief period and particularly during the waning period. Mordin et al similarly described the QoL burden of CD and subsequent improvement in QoL corresponding with symptom relief achieved with abobotulinumtoxinA treatment. ${ }^{3}$ In this study, QoL was assessed with the Medical Outcomes Study Short-Form (36-item) Health Survey (SF-36) at baseline and 8 weeks posttreatment, with significant improvements across the majority of SF-36 domains examined at the 8 -week time point. ${ }^{3}$ However, this time point is at least 4 weeks before reinjection is recommended. ${ }^{18}$ An open-label study of the QoL impact of abobotulinumtoxinA injection measured using the Craniocervical Dystonia Questionnaire (CDQ-24) - in a cohort of patients with CD who were naïve to BoNT found that treatment significantly improved total scores on the CDQ-24 at week 4, which was maintained at week 12 even though clinical measures of disease severity (Tsui scale) were deteriorating at this time point. ${ }^{25}$ However, a survey of patients who received onabotulinumtoxinA or abobotulinumtoxin A injections for $\mathrm{CD}$ found that many patients would prefer shorter treatment intervals than they currently receive..$^{14}$ It is evident that a greater understanding of the impact of BoNT injection on QoL in the real-life setting is needed. It may be that the QoL burden of CD symptom recurrence during the waning period subsequent to BoNT injection is underestimated, as QoL analyses in a clinical trial setting are performed well in advance of the need for reinjection and possibly before recurring symptoms have had time to influence QoL.

We found that symptom progression among the participants in our study was most frequently represented graphically by a bell-shaped pattern. A recently performed follow-up study (aiming to support the development of the "MyDystonia" patient app, www.mydystonia.com) used a mobile diary to further explore QoL and disease burden between BoNT injections in patients with dystonia. The outputs generally confirmed that the most frequent impact pattern was bell shaped (data not shown). Enabling patients to monitor the development of their symptoms over time between injections may help health care professionals understand their individual disease pattern and define a personalized treatment approach according to the individual needs of the patient. However, many health care professionals already acknowledge the need for individualized treatment, but are hampered by issues such as excessive workload, restrictions based on regulatory labels for BoNT formulations, and insufficient reimbursement of BoNT injections.

Given the far-reaching impact of recurring symptoms, it would be prudent to consider measures to mitigate or reduce their severity. In particular, our study revealed that patients would like BoNT therapy to enable them to experience "more good days" when the symptoms are less severe and the impact on their lives is low. One approach to achieving improved outcomes for patients would be to provide more individualized flexible and/or shorter intervals between BoNT treatments to those who require it. The responses from the participants in our study generally support this strategy. However at present, clinical trial data confirming this are scarce as most studies of botulinum toxin formulations preclude reinjection at intervals shorter than 12 weeks. Incobotulinumtoxin A is the only formulation studied in clinical trials allowing flexible dosing intervals. These studies showed that incobotulinumtoxinA for the treatment of CD can be effective and well tolerated when administered using flexible schedules with minimum injection intervals of 6 weeks ${ }^{26,27}$ or 10 weeks. ${ }^{28}$ Importantly, in these studies incobotulinumtoxinA injections were administered on the basis of clinical need and following 
physical and neurological examinations by the investigator, who assessed disease severity using clinical rating scales. Although patients were able to approach their clinic to request reinjection, this was only done after confirmation of a clinical need by the treating physician.

Several barriers would need to be overcome if such a strategy were implemented. From a regulatory perspective, authorities would need to be convinced of the safety of reducing the injection interval, and patients would also require reassurance that they are not placed at increased risk of harm from receiving BoNT injections at shorter intervals. Patient education and home-injection services may help to allay such fears. However, it is clear that patient satisfaction with BoNT treatment currently falls short of the ideal situation. Notably, in an international interview-based study exploring satisfaction with BoNT treatment among 136 patients with $\mathrm{CD}, \sim 45 \%$ of patients reported a preference for an injection interval of $\leq 10$ weeks, but only $4.4 \%$ of patients received such intervals. ${ }^{14}$ Surveyed patients had received BoNT for $>3.5$ years, with a mean interval between injection cycles of 14 weeks; the majority of patients $(42.7 \%)$ had received reinjection every $11-12$ weeks. ${ }^{14}$ This study shows an apparent need to determine the optimal treatment schedule that is tailored to each patient.

The qualitative-exploratory methodology of this study has inherent strengths and weaknesses. The study design incorporates the facility for patients to openly discuss their views and needs, thus providing real insight into the problems of living with CD on a day-to-day basis, which would go unnoticed in most other research settings.

\section{Conclusion}

Recurrence of primary symptoms of CD between BoNT injections and the secondary symptoms associated with these have a substantial impact on the QoL and well-being of patients with CD. A potential approach to optimize treatment may be personalized and flexible BoNT injection intervals, but several challenges need to be overcome before this strategy could be implemented in clinical practice. A collaborative effort between patients, clinicians, and health care providers may be one avenue to effect change and improve the daily lives of patients with CD. Such collaboration, perhaps aided by a detailed individual assessment of patients' daily symptoms, could improve the communication of individual patients' needs to their clinicians.

\section{Acknowledgments}

This study was conducted by Opinion Health, London, UK, and was supported by Merz Pharmaceuticals GmbH (Frankfurt am Main, Germany). Editorial support was provided by Rhian Harper Owen on behalf of Complete Medical Communications and funded by Merz Pharmaceuticals $\mathrm{GmbH}$.

\section{Author contributions}

All authors participated in the design of the study, and the analysis and interpretation of data. All authors critically reviewed and revised the manuscript and approved the final version for submission.

\section{Disclosure}

MP and XL are employees of Opinion Health, London, UK. MK is an employee of Merz Pharmaceuticals GmbH. The authors report no other conflicts of interest in this work.

\section{References}

1. Albanese A, Bhatia K, Bressman SB, et al. Phenomenology and classification of dystonia: a consensus update. Mov Disord. 2013;28(7): 863-873.

2. National Institutes of Health. The Dystonias. NIH Publication No 12 717. 2012. Available from: http://www.ninds.nih.gov/disorders/ dystonias/dystonias_brochure_508comp.pdf. Accessed December 18, 2015.

3. Mordin M, Masaquel C, Abbott C, Copley-Merriman C. Factors affecting the health-related quality of life of patients with cervical dystonia and impact of treatment with abobotulinumtoxinA (Dysport): results from a randomised, double-blind, placebo-controlled study. BMJ Open. 2014;4(10):e005150.

4. Pekmezovic T, Svetel M, Ivanovic N, et al. Quality of life in patients with focal dystonia. Clin Neurol Neurosurg. 2009;111(2):161-164.

5. Slawek J, Friedman A, Potulska A, et al. Factors affecting the healthrelated quality of life of patients with cervical dystonia and the impact of botulinum toxin type A injections. Funct Neurol. 2007;22(2): 95-100.

6. Molho ES, Agarwal N, Regan K, Higgins DS, Factor SA. Effect of cervical dystonia on employment: A retrospective analysis of the ability of treatment to restore premorbid employment status. Mov Disord. 2009; 24(9):1384-1387.

7. Albanese A, Asmus F, Bhatia KP, et al. EFNS guidelines on diagnosis and treatment of primary dystonias. Eur J Neurol. 2011;18(1):5-18.

8. Novak I, Campbell L, Boyce M, Fung VS. Botulinum toxin assessment, intervention and aftercare for cervical dystonia and other causes of hypertonia of the neck: international consensus statement. Eur J Neurol. 2010;17(Suppl 2):94-108.

9. Simpson DM, Blitzer A, Brashear A, et al. Assessment: botulinum neurotoxin for the treatment of movement disorders (an evidence-based review): report of the Therapeutics and Technology Assessment Subcommittee of the American Academy of Neurology. Neurology. 2008;70(19): 1699-1706.

10. Comella CL, Jankovic J, Truong DD, Hanschmann A, Grafe S; U.S.XEOMIN Cervical Dystonia Study Group. Efficacy and safety of incobotulinumtoxinA (NT 201, XEOMIN ${ }^{\circledR}$, botulinum neurotoxin type A, without accessory proteins) in patients with cervical dystonia. J Neurol Sci. 2011;308(1-2):103-109.

11. Greene P, Kang U, Fahn S, Brin M, Moskowitz C, Flaster E. Doubleblind, placebo-controlled trial of botulinum toxin injections for the treatment of spasmodic torticollis. Neurology. 1990;40(8):1213-1218.

12. Truong D, Duane DD, Jankovic J, et al. Efficacy and safety of botulinum type A toxin (Dysport) in cervical dystonia: results of the first US randomized, double-blind, placebo-controlled study. Mov Disord. 2005;20(7):783-791. 
13. Dressler D, Tacik P, Adib Saberi F. Botulinum toxin therapy of cervical dystonia: comparing onabotulinumtoxinA (Botox $\left.{ }^{\circledR}\right)$ and incobotulinumtoxinA (Xeomin ${ }^{\circledR}$ ). J Neural Transm (Vienna). 2014;121(1):29-31.

14. Sethi KD, Rodriguez R, Olayinka B. Satisfaction with botulinum toxin treatment: a cross-sectional survey of patients with cervical dystonia. J Med Econ. 2012;15(3):419-423.

15. Marsh WA, Monroe DM, Brin MF, Gallagher CJ. Systematic review and meta-analysis of the duration of clinical effect of onabotulinumtoxinA in cervical dystonia. BMC Neurol. 2014;14:91.

16. Dressler D, Tacik P, Saberi FA. Botulinum toxin therapy of cervical dystonia: duration of therapeutic effects. J Neural Transm. 2015;122(2): 297-300.

17. Allergan. Botox ${ }^{\circledR} 100 \mathrm{U}$ summary of product characteristics [updated March 30, 2015]. Available from: http://www.medicines.org.uk/EMC/ medicine/112/SPC/. Accessed December 18, 2015.

18. Ipsen. Dysport ${ }^{\circledR} 300 \mathrm{U}$ and $500 \mathrm{U}$ summary of product characteristics [updated September 16, 2015]. Available from: http://www.medicines. org.uk/EMC/medicine/870/SPC/. Accessed December 18, 2015.

19. Merz Pharma UK Ltd. XEOMIN ${ }^{\circledR} 100$ U summary of product characteristics [updated June 30, 2014]. Available from: http://www.medicines. org.uk/emc/medicine/20666. Accessed December 18, 2015.

20. Greene P, Fahn S, Diamond B. Development of resistance to botulinum toxin type A in patients with torticollis. Mov Disord. 1994;9(2): 213-217.

21. Albanese A, Abbruzzese G, Dressler D, et al. Practical guidance for CD management involving treatment of botulinum toxin: a consensus statement. J Neurol. 2015;262(10):2201-2213.
22. Fernandez HH, Pagan F, Danisi F, et al; XCiDaBLE Study Group. Prospective study evaluating incobotulinumtoxinA for cervical dystonia or blepharospasm: Interim results from the first 145 subjects with cervical dystonia. Tremor Other Hyperkinet Mov (N Y). 2013;3.

23. Werle RW, Takeda SY, Zonta MB, Guimaraes AT, Teive HA. The physical, social and emotional aspects are the most affected in the quality of life of the patients with cervical dystonia. Arq Neuropsiquiatr. 2014;72(6):405-410.

24. Hallett M, Albanese A, Dressler D, et al. Evidence-based review and assessment of botulinum neurotoxin for the treatment of movement disorders. Toxicon. 2013;67:94-114.

25. Hefter H, Benecke R, Erbguth F, Jost W, Reichel G, Wissel J. An openlabel cohort study of the improvement of quality of life and pain in de novo cervical dystonia patients after injections with $500 \mathrm{U}$ botulinum toxin A (Dysport). BMJ Open. 2013;3(4).

26. Evidente VG, Fernandez HH, LeDoux MS, et al. A randomized, doubleblind study of repeated incobotulinumtoxinA $\left(\mathrm{Xeomin}^{\circledR}\right)$ in cervical dystonia. J Neural Transm (Vienna). 2013;120(12):1699-1707.

27. Evidente VG, Truong D, Jankovic J, Comella CL, Grafe S, Hanschmann A. IncobotulinumtoxinA (Xeomin ${ }^{\circledR}$ ) injected for blepharospasm or cervical dystonia according to patient needs is well tolerated. J Neurol Sci. 2014;346:116-120.

28. Dressler D, Paus S, Seitzinger A, Gebhardt B, Kupsch A. Long-term efficacy and safety of incobotulinumtoxinA injections in patients with cervical dystonia. J Neurol Neurosurg Psychiatry. 2013;84(9): 1014-1019.
Patient Preference and Adherence

\section{Publish your work in this journal}

Patient Preference and Adherence is an international, peer-reviewed, open access journal that focuses on the growing importance of patient preference and adherence throughout the therapeutic continuum. Patient satisfaction, acceptability, quality of life, compliance, persistence and their role in developing new therapeutic modalities and compounds to optimize

\section{Dovepress}

clinical outcomes for existing disease states are major areas of interest for the journal. This journal has been accepted for indexing on PubMed Central. The manuscript management system is completely online and includes a very quick and fair peer-review system, which is all easy to use. Visit http://www. dovepress.com/testimonials.php to read real quotes from published authors. 